\title{
Identification of dynamic models for a wave energy converter from experimental data
}

\author{
Simone Giorgi ${ }^{\mathrm{a}, *}$, Josh Davidson ${ }^{\mathrm{a}}$, Morten Jakobsen ${ }^{\mathrm{b}}$, Morten Kramer ${ }^{\mathrm{b}}$, John V. Ringwood ${ }^{\mathrm{a}}$ \\ a Center for Ocean Energy Research, Maynooth University, Maynooth, Co. Kildare, Ireland \\ ${ }^{\mathrm{b}}$ Dept of Civil Eng., Aalborg University, Denmark
}

\section{A R T I C L E I N F O}

\section{Keywords:}

Wave energy

System identification

Wave tank test

Discrete-time modelling

Nonlinear

NARX model

ARX model

Kolmogorov-Gabor polynomial model

\begin{abstract}
A B S T R A C T
This paper addresses the issue of hydrodynamic model identification from recorded tank test data, for a prototype wave energy device. The study focusses on nonlinear Kolmogorov-Gabor polynomial models, with linear models also used as a baseline reference. Six different experimental data sets are employed for model identification and validation, all derived from a JONSWAP input sea state. Compared to identification on numerical data, this study shows that the determination of model structure and orders is not so straightforward, but that consistent and useful computationally efficient models can be obtained. For the particular tests undertaken, in which the prototype device generally behaves as a wave follower, the nonlinear models only show very marginal performance improvement over the linear ones.
\end{abstract}

\section{Introduction}

Mathematical modelling of wave energy converters (WECs) has many uses, including simulation of device motion, optimisation of the WEC geometric shape, power production assessment and as a basis for model-based control design. In all these cases, it is important to describe how the body moves in the water, interacting with the waves propagating on the fluid surface. The hydrodynamic laws (mass, momentum and energy conservation laws) are the foundation for a complete description of the WEC-fluid interaction, but their solution represents a very complex and challenging problem. Different approaches to WEC-fluid interaction modelling, such as computational fluid dynamics (CFD) and linear potential theory (LPT), lead to different mathematical models, each one characterised by different accuracy and computational speed. It is crucial to realise that a mathematical model is always an approximation of the natural world, and a model that better describes the reality is not necessarily the best model; the assessment of a model depends on its purpose and application (Pintelon and Schoukens, 2012). Indeed, on one side, fully nonlinear CFD models, based on hydrodynamic laws, are able to describe the full range of hydrodynamic effects, but are very computationally expensive; for example, in three-dimensional numerical wave tanks (NWTs), $1 \mathrm{~h}$ of simulation would require approximately $1000 \mathrm{~h}$ of computation (42 days!) (Davidson et al., 2016). Therefore, the high accuracy of CFD models is not sufficient to justify their use in all WEC model applications. Indeed, the use of CFD models, in order to perform seasonal WEC power production calculations, would require decades of computation. Furthermore, a WEC mathematical model, utilised for model-based control, has to be able to produce an output simulation in real-time, certainly beyond the CFD possibility. On the other hand, LPT models (which represent the most common state-of-the-art for WEC modelling) are based on the assumptions of inviscid fluid, irrotational flow, small waves and small body motion, which completely remove the hydrodynamic nonlinearity of the WEC-fluid interaction. Linear models have good computational speed, but are not able to properly describe nonlinear hydrodynamic effects, such as nonlinear restoring force, viscosity, nonlinear excitation force, vortex shedding, which are relevant in some WEC power production conditions, since WECs are designed to operate over a wide range of wave amplitudes, and experience large motions. Therefore, it is desirable to develop hydrodynamic models with characteristics that lie somewhere between the CFD and LPT computational/accuracy extremes; ideally, a good compromise able to describe the most important nonlinearities of the real system, without requiring excessive computational time (Giorgi et al., 2016b; Penalba et al., 2017; Wolgamot and Fitzgerald, 2015).

One way to construct a mathematical model of a process under investigation involves breaking the system into subsystems and, by applying the appropriate physical laws, building up a mathematical description between the key variables. In the case of highly complex systems, the procedure may take an unacceptable amount of time and

\footnotetext{
* Corresponding author.

E-mail addresses: giorgi.simon@gmail.com (S. Giorgi), jdavidson@eeng.nuim.ie (J. Davidson), mmj@civil.aau.dk (M. Jakobsen), mmk@civil.aau.dk
} (M. Kramer), John.Ringwood@nuim.ie (J.V. Ringwood). 


\begin{tabular}{|ll|}
\hline Nomenclature & \\
ARX & Autoregressive with exogenous input \\
CFD & Computational fluid dynamics \\
DT & Discrete-time \\
FSE & Free surface elevation \\
KGP & Kolmogorov-Gabor polynomial \\
KGP $(p)$ & KGP model with maximum polynomial \\
& order $p$ \\
LF & Loss function \\
LPT & Linear potential theory \\
MAPE & Mean absolute percentage error \\
MSE & Mean squared error \\
NARX & Nonlinear autoregressive with exogenous \\
& input \\
NRMSE & Normalised root mean-squared error \\
NWT & Numerical wave tanks \\
PTO & Power take off \\
RWT & Real wave tank \\
SI & System identification \\
WEC & Wave energy converter \\
& \\
\hline
\end{tabular}

may provide a very complicated model, which could be difficult to utilise. It may also be difficult to accurately parameterise such models. This paper utilises an alternative pragmatic framework for hydrodynamic model construction, based on wave energy recorded data and system identification (SI) techniques.

The use of SI techniques in WEC modelling is not a novelty, they have been applied by some researchers to add various specific nonlinear terms to linear hydrodynamic models, in order to improve the model accuracy. In (Zurkinden et al., 2014), a nonlinear hydrostatic restoring term, identified from real wave tank (RWT) data, is introduced into the numerical model. In (Paparella et al., 2016), a viscous matrix is identified from RWT data, in order to improve the linear model describing a three-body hinge-barge device. In (Jakobsen et al., 2016) and (Zurkinden et al., 2014), a nonlinear viscous term is identified from RWT and introduced into the numerical model of a hemispherical WEC. In (Folley et al., 2007), the dynamics of small seabed-mounted bottomhinged WECs (which have the same physical principle of EB Frond, WaveRoller, Oyster and BioWave WECs) are studied, by introducing a nonlinear viscous term identified from RWT data. In (Bhinder et al., 2011, 2012) (Bhinder, 2013) a viscous term is identified from NWT, in order to describe the dynamics of floating and surging WECs. In (Atluri et al., 2009), the drag coefficient of a floating plate, moving in heave, is estimated by utilising NWT experiments. In (Giorgi and Ringwood, 2017), different drag coefficients are identified from NWT data, in the case of a floating heaving point absorber. Other studies have extended the use of SI techniques from the identification of specific nonlinear hydrodynamic terms to the whole linear/nonlinear hydrodynamic model, by utilising NWT data (Davidson et al., 2015, 2016) (Ringwood et al., 2016) (Giorgi et al., 2016a; Giorgi, 2017) (Armesto et al., 2014). The passage from NWT data to RWT data, for the identification of whole linear hydrodynamic models, can be found in (Bacelli et al., 2017) (Coe et al., 2016). In (Cho et al., 2018), a whole nonlinear hydrodynamic Hammerstein-Wiener model is identified from RWT data. In this work, whole hydrodynamic models, based on a Kolmogorov-Gabor polynomial (KGP) structure, are identified from RWT data.

The SI framework is characterised by an iterative sequence of four steps, i.e. experimental data gathering, fitting criterion and identification algorithm selection, model selection (model structure selection and model order selection), and model validation, as shown in Fig. 1. At the end of the SI procedure, a hydrodynamic parametric model is provided.

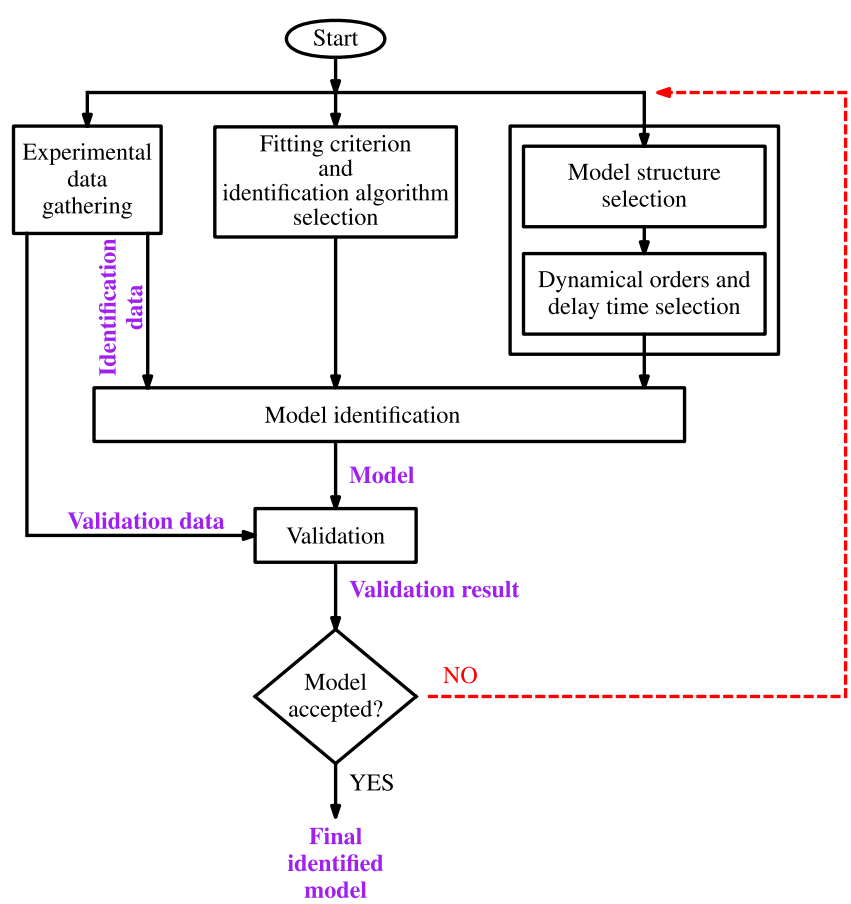

Fig. 1. Closed-loop block diagram to identify models from recorded data.

In the wave energy community, the two main sources of experimental device data are simulated experiments generated in a NWT, and real experiments generated in a RWT. All NWTs are implemented by utilising mathematical models, which necessarily are an approximation of the real system under study (e.g. the approximations introduced from the turbulence model). Furthermore, the approximated equations utilised are resolved with numerical techniques, which introduce additional numerical errors. As a result, a NWT simulation does not completely correspond to the time-evolution of the real system. On the other hand, a RWT allows the observation and study of the real system, without the introduction of any modelling error. However, in the RWT case, measurement errors are present. See (Davidson et al., 2016) (Giorgi, 2017) (Ringwood et al., 2016) for more details regarding advantages and disadvantages of NWT and RWT experiments.

The Wavestar WEC, investigated in this paper, is a well known device within the wave energy community, and has been repeatedly studied in recent years. Different kinds of models have been utilised to describe its behaviour, some of them purely linear, based on the assumption of linear potential theory (Hansen and Kramer, 2011) and, more recently, nonlinear terms have been introduced into the models. In (Zurkinden et al., 2014), a nonlinear hydrostatic restoring moment, described by a cubic polynomial function, is introduced into the model. In (Zurkinden et al., 2014) and (Jakobsen et al., 2016), a drag term is introduced by utilising Morison's equation. The accuracy of these nonlinear WEC models show some improvement, over their linear counterparts, especially for peak displacement but, at the same time, viscous drag moments may not be manifested in the case of a simple passively damping power take off (PTO) (Zurkinden et al., 2014). (Giorgi and Ringwood, 2016) examines the influence of the nonlinear Froude-Krylov force on the dynamics of the scaled Wavestar WEC utilised in this paper.

The remainder of paper is laid out as follows; Section 2 explains the RWT experiments used to produce the system identification data, and Section 3 describes the process of identifying the model parameters from the RWT gathered data. In Section 4, the structure of the utilised linear and nonlinear models is outlined, while Section 5 describes the salient points of model validation. Section 6 shows the results of the proposed procedure for the case of the scaled Wavestar WEC and, finally, conclusions are drawn in Section 7. 


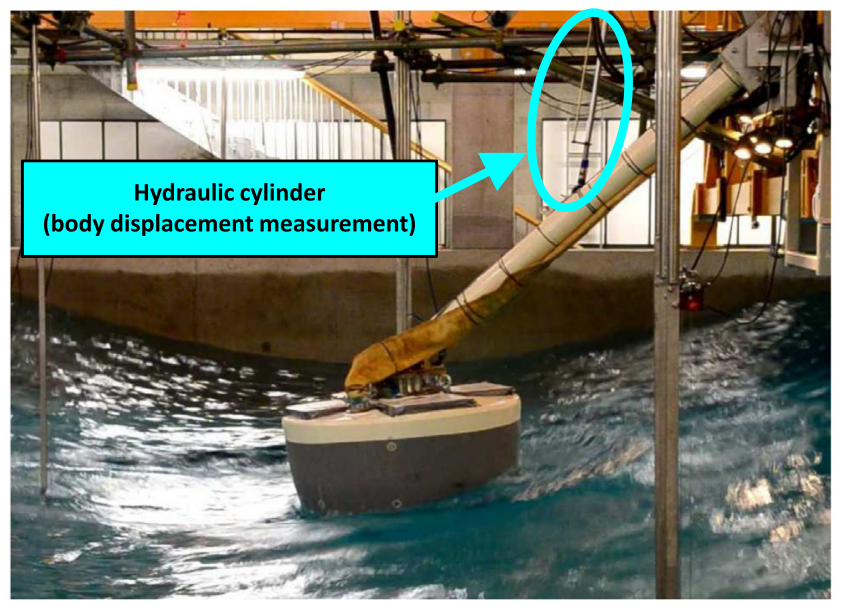

Fig. 2. Scaled Wavestar WEC tested at Plymouth. The arrow shows the hydraulic cylinder used to measure the body displacement and to apply, during the tank experiments, the PTO force described by Eq. (1).

\section{Wave tank experimental data gathering}

\subsection{Wave tank facility specifications}

The data utilised for the current paper have been collected during a period of four weeks in September 2013, through the MARINET access program at the COAST Laboratory of Plymouth University, UK (COAST, 2018). The RWT is $35 \mathrm{~m}$ long by $15.5 \mathrm{~m}$ wide and with an adjustable depth floor, which can be set with a depth up to $3 \mathrm{~m}$ (the data utilised in this paper were recorded with a $3 \mathrm{~m}$ depth floor). At one end of the wave tank, 24 dry-backed flap wavemakers of $2.0 \mathrm{~m}$ hinge depth are installed, each of them individually controlled for wave generation. The presence of an absorbing beach, at the other end of the wave tank, significantly reduces the amplitude of reflected waves.

\subsection{Device specifications}

The scaled Wavestar WEC studied is a point-absorber composed of a hemispherical float having a diameter of about $1 \mathrm{~m}$, rigidly attached to a $2 \mathrm{~m}$ length arm, as shown in Fig. 2 (Jakobsen, 2015, 2014). The total weight of the float-arm system is $130 \mathrm{~kg}$. The WEC is attached to the bridge at the facility via a mounting frame. The WEC is constrained to have a single degree of freedom: the rigid float-arm structure rotates in pitch around a fixed hinge, located $1.69 \mathrm{~m}$ above the mean free surface elevation (FSE). A hydraulic cylinder is attached to the support frame, roughly halfway down the arm (see Fig. 2), with the purpose of applying a PTO force, $f_{c y l}$, described by:

$f_{c y l}=-K_{c y l} \Delta_{c y l}-D_{c y l} \dot{\Delta}_{c y l}$

where $K_{c y l}=50 \mathrm{~N} / \mathrm{m}, D_{c y l}=100 \mathrm{Ns} / \mathrm{m}$ are the stiffness and damping coefficients, respectively, utilised in the tank experiments, $\Delta_{c y l}$ is the hydraulic cylinder displacement and $\dot{\Delta}_{c y l}$ is the hydraulic cylinder velocity. The WEC displacement is measured as the extension of the hydraulic cylinder (Jakobsen, 2014), where $\Delta_{c y l}$, measured by the sensor, is positive when the cylinder extends and, consequently, when the float enters into the water. However, it is desirable having a variable, representing the body displacement, which is positive when the float emerges from the water and negative when entering the water; therefore, the body displacement is conventionally defined as $y=-\Delta_{c y l}$.

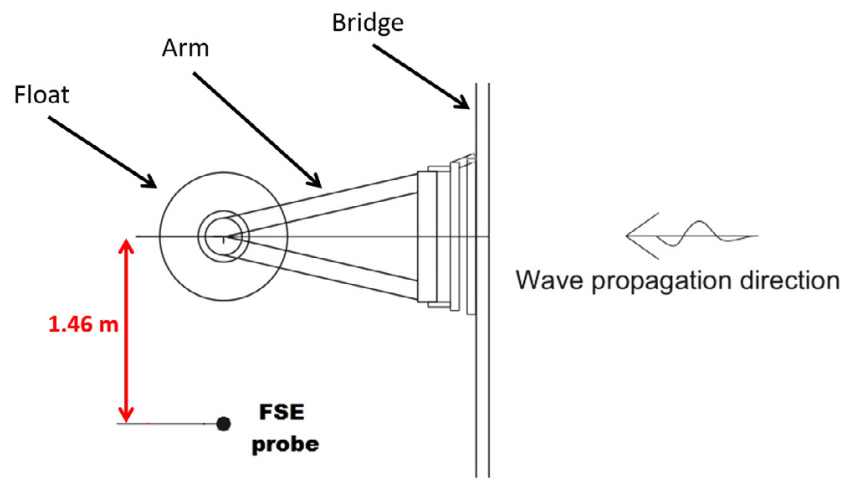

Fig. 3. Relative position of the WEC and the FSE probe (top view) (Jakobsen, 2014).

\begin{tabular}{|c|c|c|}
\hline Exp. name & $H_{s}[\mathrm{~m}]$ & $T_{p}[\mathrm{~s}]$ \\
\hline$E_{1}$ & 0.124 & 2 \\
\hline$E_{2}$ & 0.184 & 2.5 \\
\hline$E_{3}$ & 0.245 & 3 \\
\hline$E_{4}$ & 0.369 & 2.5 \\
\hline$E_{5}$ & 0.490 & 3 \\
\hline$E_{6}$ & 0.720 & 4 \\
\hline
\end{tabular}

\subsection{Free surface elevation characteristics}

The input variable, utilised for the mathematical model, is the FSE available at the center of mass of the float, the measurement of which is problematic, since the body occupies that position. This problem is overcome by measuring the FSE at the side of the float, on a line parallel to the wavefront and passing through the center of mass of the float, as shown in Fig. 3. In this way, it is possible to obtain the FSE elevation at the center of mass of the float (plus the waves radiated by the body). Six different RWT experiments are utilised for the WEC modelling (i.e. $E_{1}, E_{2}, E_{3}, E_{4}, E_{5}$ and $E_{6}$ ). The FSE of each experiment is a realization of different irregular sea states characterised by a JONSWAP spectrum with peakiness factor $\gamma=3.3$. The significant wave height, $H_{s}$, and peak wave period, $T_{p}$, of the different experiments are shown in Table 1. In Fig. 4, it is possible to see the time evolution of the FSE and body displacement for experiment $E_{1}$.

The nonlinear interaction between the body and the water is strongly dependent on the extent of the time-varying wetted body surface. Since the body displacement alone is not sufficient to provide an indication of the variation in the wetted body surface, it is more useful to take into consideration the relative float heave displacement, $y_{r h}$, defined as $y_{r h}=y_{h}-\eta$, where $y_{h}$ is the float heave displacement and $\eta$ the FSE at the center of mass of the float. Indeed, $y_{r h}$ provides a more accurate measure of the amount of the body that is submerged into the water. By using the geometric information from the float-arm system, it is possible to convert the hydraulic cylinder displacement, $\Delta_{c y l}$, into the heave float displacement $y_{h}$. Fig. 5 displays the relative float heave displacement for experiment $E_{1}$. Fig. 5(a) shows the relative float heave displacement time evolution, while Fig. 5(b) shows the relative float heave displacement probability distribution.

In the six experiments utilised in this paper, the relative float heave displacement $y_{r h}$ is, for the majority of the time, contained between -0.1 and $+0.1 \mathrm{~m}$, as shown in Figs. 5 and 6 . The reduced $y_{r h}$ (compared to the vertical float dimension) is a consequence of the applied control strategy, described by Eq. (1), and of the values of the PTO coefficients $K_{c y l}$ and $D_{c y l}$, utilised for the tank tests, which result in the 'wave following' behaviour of the device. In the case of $y_{r h}=-0.1 \mathrm{~m}$, the body cross-section has a radius of $0.467 \mathrm{~m}$, which corresponds to a 

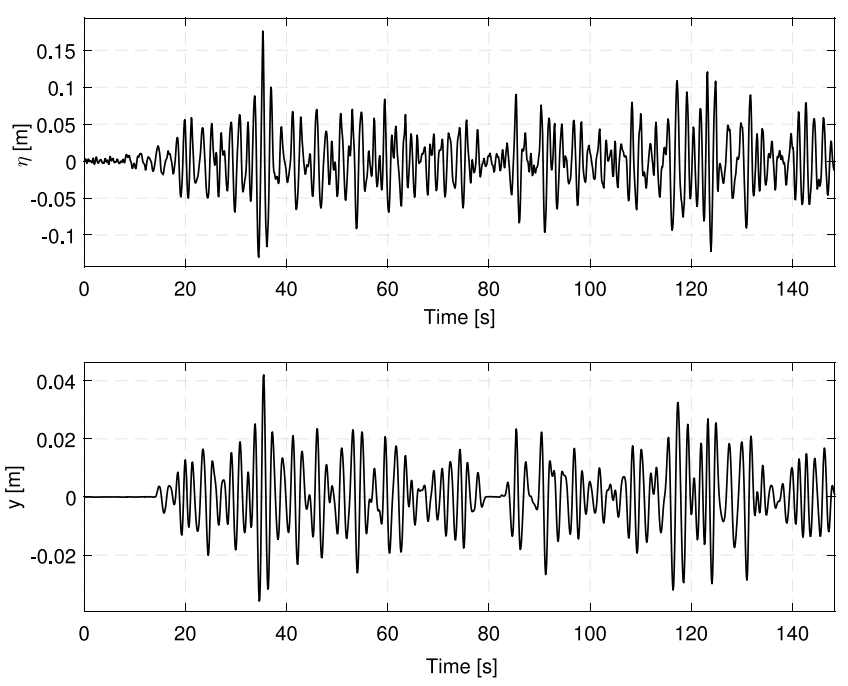

Fig. 4. Signals time evolution for experiment $E_{1}$. (a)

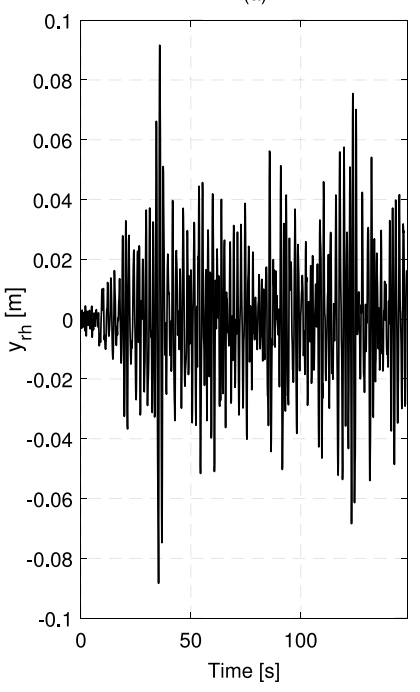

(b)

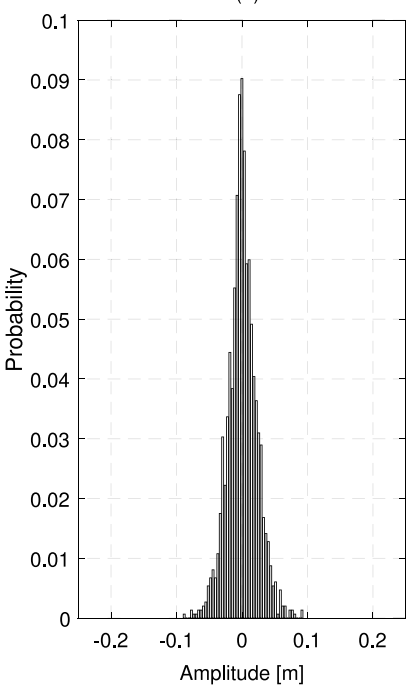

Fig. 5. The body displacement alone does not provide a sufficient indication of the variation in the wetted body surface, it is more useful to take into consideration the relative float heave displacement $y_{r h}=y_{h}-\eta$. (a) Time evolution of $y_{r h}$ for experiment $E_{1}$. (b) Probability distribution of $y_{r h}$ for experiment $E_{1}$.

cross-sectional area $A_{1}=0.685 \mathrm{~m}^{2}$. On the other hand with $y_{r h}=$ $0.1 \mathrm{~m}$, the cross-section has a radius of $0.502 \mathrm{~m}$, which corresponds to a cross-sectional area $A_{2}=0.792 \mathrm{~m}^{2}$. Fig. 6(b) shows the two cross-sectional areas in the case of $y_{r h}$ equal to 0.1 and -0.1. The relationship $A_{1} / A_{2}=0.87$, which represents a rare extreme case in the six experiments utilised, suggests that the average hydrodynamic nonlinearities involved with the change of the cross-sectional area are modest. Indeed, for a heaving body, if the cross-sectional area is uniform, the restoring force has a linear relationship with the body displacement (the restoring coefficient is given by $K=\rho g A$, where $\rho$ is the water density, $g$ the gravitational acceleration and $A$ the constant cross-sectional area) (Falnes, 2002).

\section{Fitting criterion and identification algorithm selection}

Given the experimental data and a parametric model structure (Norton et al., 2010), characterised by the parameter vector $\boldsymbol{\theta}$, it is necessary to identify some parameter values $\hat{\theta}$, in order to obtain the 'best' model

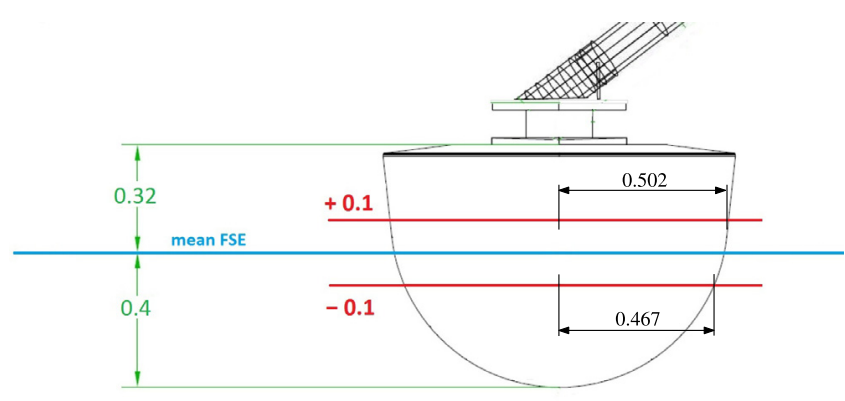

(a)

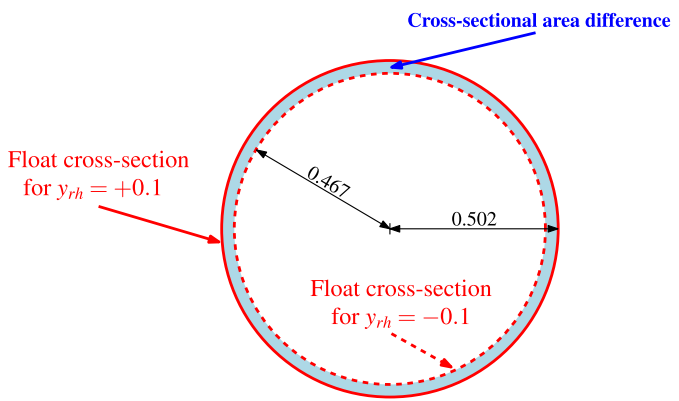

(b)

Fig. 6. In the six experiments utilised in this paper, the relative float heave displacement is, for the majority of the time, contained between -0.1 and $+0.1 \mathrm{~m}$, which corresponds to a circular cross-section with radius between 0.467 and $0.502 \mathrm{~m}$. (a) Geometric dimensions of the float in metres (side view). (b) Float cross-sections (top view).

able to describe the data. A way to quantify the model performance is provided by the use of a loss function (LF), $J(\theta)$, (also denoted fitting criterion or error metric), between the experimental data and the model prediction. Therefore, given the experimental data, a model structure, and a LF, each parameter vector value produces a singular scalar number, which represents the model performance. One of the most common and utilised LFs is the mean squared error (MSE) (Ljung, 1999). By changing all possible parameter values, $J$ describes a multidimensional surface (in general multimodal), having a global minimum and multiple local minima. The objective of the identification algorithm is to find the parameter values $\hat{\theta}$ which minimise $J$, in order to identify the best model able to fit the experimental data. If the model output is a linear function of $\theta$ and the MSE is used as a fitting criteria, then $J(\theta)$ is a quadratic function with an unique minimum and an analytical solution exists (linear optimisation), given by:

$\hat{\boldsymbol{\theta}}=\left(\boldsymbol{\Phi}^{\mathrm{T}} \boldsymbol{\Phi}\right)^{-1} \boldsymbol{\Phi}^{\mathrm{T}} \mathbf{y}$

where $\boldsymbol{\Phi}$ is the regression matrix and $\mathbf{y}$ the observation vector (Soderstrom and Stoica, 1989) (Draper and Smith, 1998) (Nelles, 2001). In this paper, the minimisation of the LF is not computed directly using Eq. (2), because the use of $\boldsymbol{\Phi}^{\mathrm{T}} \boldsymbol{\Phi}$ increases the possibility of obtaining an ill-conditioned problem (Ljung, 1999) (Norton, 2009) (Sauer, 2012). Instead, the regression matrix is factorised as $\boldsymbol{\Phi}=Q R$, where $Q$ is an orthogonal square matrix and $R$ an upper triangular matrix (QR factorisation method). In this way, the least squares solution is computed directly from $\boldsymbol{\Phi}$, without forming $\boldsymbol{\Phi}^{\mathrm{T}} \boldsymbol{\Phi}$ (Golub and Van Loan, 2012) (Sauer, 2012) (Giorgi, 2017). On the other hand, if the model output is nonlinear in $\theta$ then, in general, $J(\theta)$ is a multimodal function; no analytical solution exists and numerical optimisation is required (a nonlinear optimisation algorithm has to be selected). We note that a model can have a nonlinear input/output relationship but be linear in the parameters. 


\section{Model selection}

The choice of a model structure, in order to obtain a correct description of the relationship between input and output data, is an important and difficult step. Adopting a SI approach offers considerable flexibility, with both linear and nonlinear potential model parametrisations, regarding the desired complexity/fidelity trade-off. The identified parametric model should (ideally) be able to capture the essential nonlinear dynamics but retain a sufficient simplicity that allows the model to be run in real time. Considering the discrete-time (DT) nature of sampled data from experiments, the majority of system identification techniques are based on discrete-time models (Ljung, 1999). Discretetime modelling uses signals only specified at the discrete time instants $t=k T_{s}$, where $T_{s}$ is the sampling period and $k$ is an integer. In this paper, nonlinear autoregressive with exogenous input (NARX) models are utilised, which are described by the following DT input/output relationship (Giorgi, 2017; Giorgi et al., 2016a) (Ringwood et al., 2016):

$$
\begin{aligned}
y(k)= & g\left[y(k-1), \ldots, y\left(k-n_{a}\right),\right. \\
& \left.\eta\left(k-n_{d}\right), \eta\left(k-n_{d}-1\right), \ldots, \eta\left(k-n_{d}-n_{b}\right)\right],
\end{aligned}
$$

where $g$ is a general function, $y$ is the body displacement, $\eta$ the FSE, $n_{a}$ and $n_{b}$ the dynamical order of the model, and $n_{d}$ the input delay index ( $T_{d}=n_{d} T_{s}$ is termed the delay time). Details about the comparison of DT and continuous-time models for wave energy applications can be found in (Giorgi, 2017). In Eq. (3), the relationship between $\eta$ and $y$ is noncausal (Falnes, 1995), therefore, a $n_{d}<0$ is expected (Giorgi, 2017; Giorgi et al., 2016a) (Ringwood et al., 2016). As shown in Fig. 1, the selection of a model is based on two main steps: model structure selection, and dynamical order and delay time selection.

\subsection{Model structure selection}

\subsubsection{ARX model structure}

The first parametric model examined is the autoregressive with exogenous input (ARX) model, for which, Eq. (3) becomes:

$y(k)=\sum_{i=1}^{n_{a}} a_{i} y(k-i)+\sum_{i=0}^{n_{b}} b_{i} \eta\left(k-n_{d}-i\right)$

It is a well-known black box model, linear in the parameters $a_{i}$ and $b_{i}$, and with a linear input/output relationship (Ljung, 1999).

\subsubsection{Nonlinear Kolmogorov-Gabor polynomial model}

The second model considered in this paper utilises a polynomial nonlinearity, yielding a KGP model (Nelles, 2001). In this case, Eq. (3) becomes:

$y(k)=\sum_{j=1}^{p}\left[\sum_{i=1}^{n_{a}} a_{i j} y^{j}(k-i)+\sum_{i=0}^{n_{b}} b_{i j} \eta^{j}\left(k-n_{d}-i\right)\right]$

where the cross-product terms (between $\eta$ and $y$ ) have been removed, since the they can lead to potential and unpredictable stability problems (Giorgi, 2017; Giorgi et al., 2016a) (Ringwood et al., 2016), and where $p$ is the maximum polynomial order for the terms involving $\eta$ and $y$ alone. The KGP model is a black box model having a nonlinear input/output relationship, but has the advantage of being linear in the parameters $a_{i j}$ and $b_{i j}$.

\subsection{Dynamical orders and delay time selection}

The dynamical order of a parametric model is related to the number of parameters utilised. By increasing the order, the model becomes more flexible and able to show more complex dynamical behaviour but, at the same time, unnecessarily high orders can make the model less able to generalise on new data (i.e. overfitting) (Box et al., 1994).

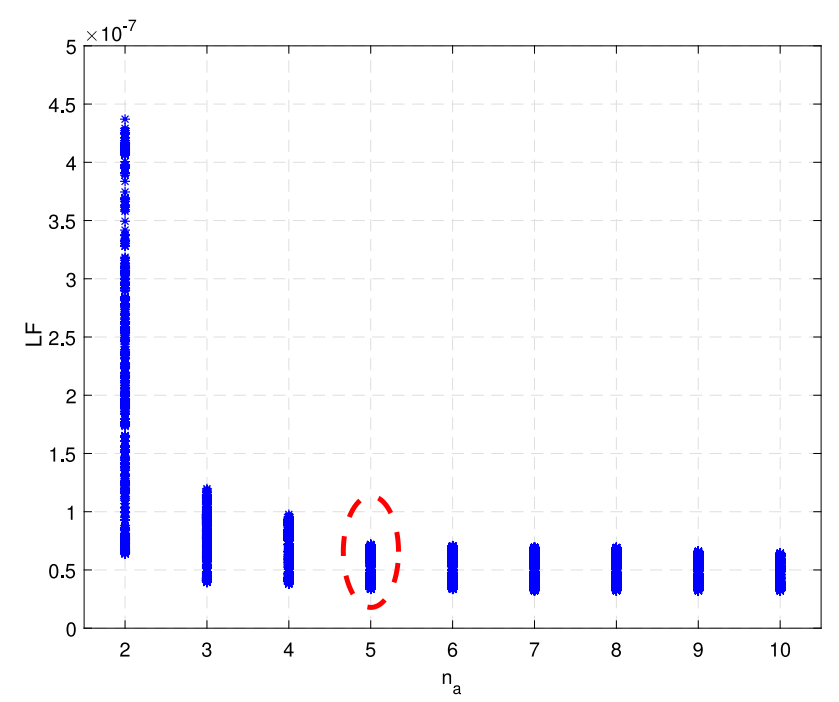

Fig. 7. Loss function for experiment $E_{1}$ (each vertical 'line' shows the results of the $310 n_{b}$ and $n_{d}$ combinations). For $n_{a}>5$ there is no relevant reduction of the LF ( $n_{a}=5$ is the correct value to obtain a parsimonious model structure).

Indeed, a model is identified by maximising its performance on a training data set but, if the model begins to 'memorise' the specifics of the particular training data segment, rather than 'learning' to generalise, the model has no value, other than being able to reproduce the training data. In general, it is necessary to determine a parsimonious model order (Soderstrom and Stoica, 1989) (Box et al., 1994) (Burnham and Anderson, 2013), which will work well with the training data and, at the same time, generalises well to other new data. Soderstrom states the parsimony principle in (Soderstrom and Stoica, 1989): 'Given two or more possible models, which all explain the data well, the model with the smallest order should be selected'.

In the case of nonlinear dynamic systems, the problem of model order determination is still not satisfactorily solved. A widely applied approach is to identify different models having increasing orders and to select the best compromise between complexity and accuracy (Nelles, 2001), An indication that a correct model order has been selected is provided by the fact that the model performance shows diminishing returns beyond a certain order (Isermann and Mnchhof, 2011). In this paper, the time delay and dynamical orders are estimated by implementing a systematic trial and error process on several (linear) ARX models (Giorgi et al., 2016a) (Keesman, 2011) (Chetouani, 2008; Ringwood et al., 1993), with varying $n_{a}, n_{b}$ and $n_{d}$, and selecting the values which give the best model performance, as measured by a loss function $L F$, which depends simultaneously on $n_{a}, n_{b}$ and $n_{d}$. In total, 3100 different ARX structures are examined utilising all the possible combinations of the integers $1 \leq n_{a} \leq 10,0 \leq n_{b} \leq 9$ and $-20 \leq n_{d} \leq 10$ (it is possible to efficiently realise this calculation with the use of the Matlab command arxstruc).

Since $n_{a}$ indicates the number of poles of the system, it is the most important parameter and is the first to be estimated. In Fig. 7, the loss function $L F=L F\left(n_{a}, n_{b}, n_{d}\right)$, for experiment $E_{1}$, is plotted versus $n_{a}$, where each vertical 'line' comprises marker points for the results of the $310 n_{b}$ and $n_{d}$ combinations $\left(0 \leq n_{b} \leq 9\right.$ and $\left.-20 \leq n_{d} \leq 10\right)$, showing the range of LF for a specific value of $n_{a}$. With an objective of minimising the LF, there is no relevant reduction in LF for $n_{a}>5$. Very similar results are obtained for experiments $E_{2}, E_{3}, E_{4}, E_{5}$ and $E_{6}$. These results indicate that $n_{a}=5$ is the correct value to obtain a parsimonious model structure.

Once $n_{a}$ is selected, the next step is the estimation of $n_{b}$ and $n_{d}$. Plotting a different LF curve versus $n_{d}$, for each value of $n_{b}$, shows that the minimum of the LF occurs at different values for $n_{d}$, depending on 


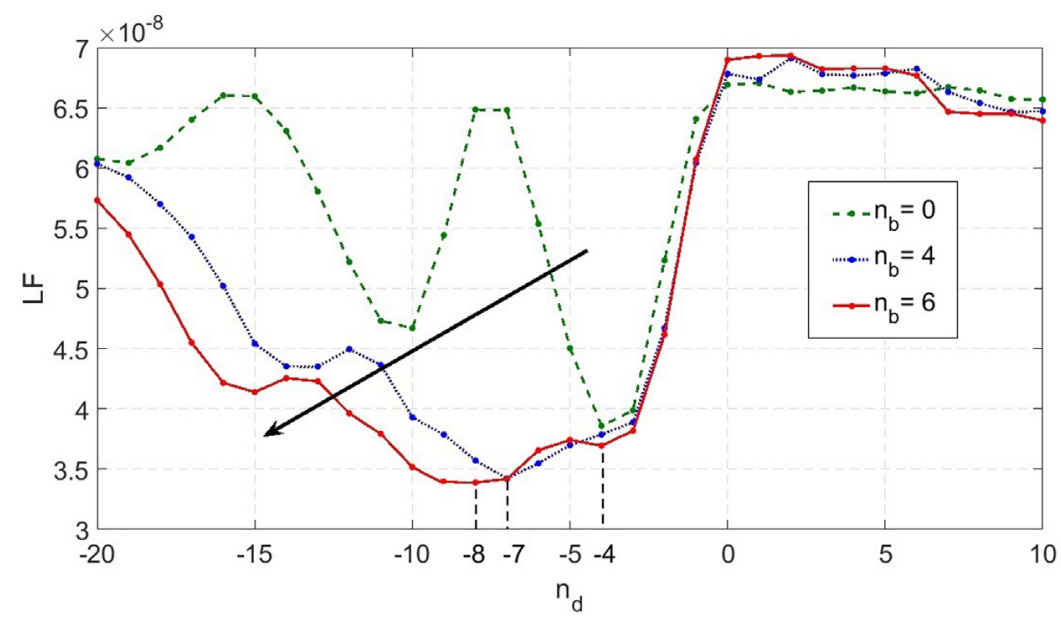

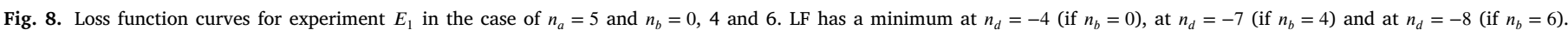
The curves move down and left with $n_{b}$ increasing from 0 to 4 to 6 , as indicated by the black arrow.

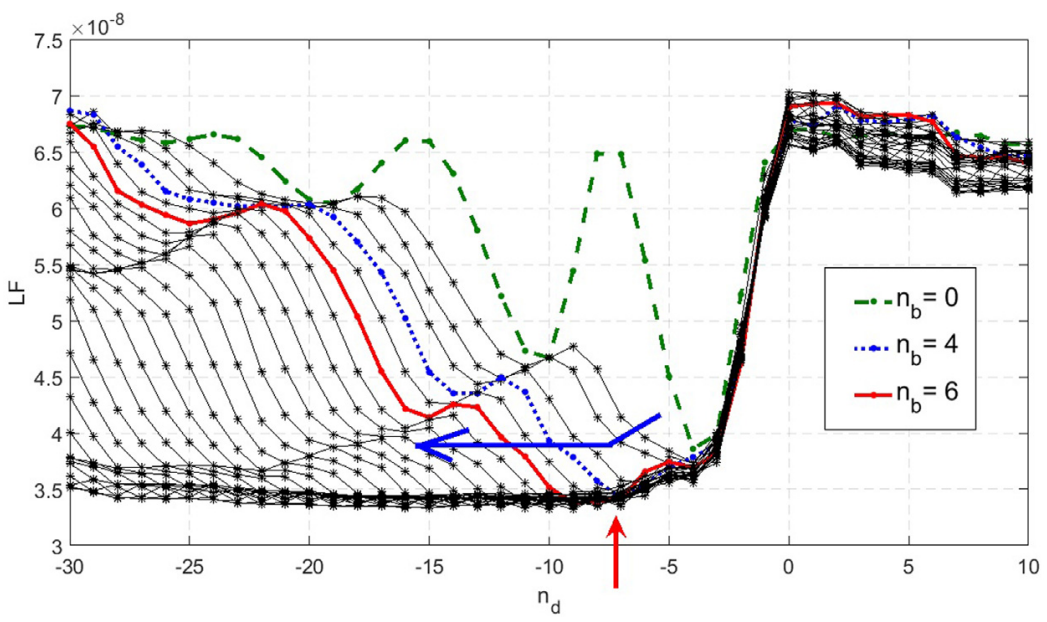

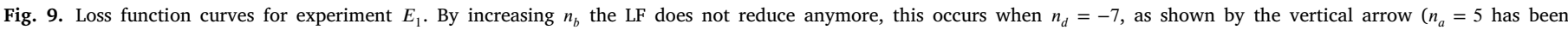

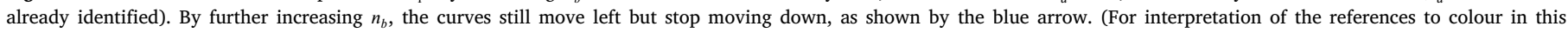
figure legend, the reader is referred to the web version of this article.)

the value of $n_{b}$. Indeed, Fig. 8 shows the LF curves for experiment $E_{1}$ for the case of $n_{a}=5$ and $n_{b}=0,4$ and 6 ; it is possible to see that LF has a minimum at $n_{d}=-4$ (if $n_{b}=0$ ), at $n_{d}=-7$ (if $n_{b}=4$ ) and at $n_{d}=-8$ (if $n_{b}=6$ ). Fig. 8 also shows that the curves move down and left as $n_{b}$ increases from 0 to 4 to 6 , as indicated by the black arrow in the picture. By further increasing $n_{b}$, the curves still move left but stop moving down, as shown by the blue arrow in Fig. 9 with $L F=L F\left(n_{a}=5,0 \leq n_{b} \leq 29,-30 \leq n_{d} \leq+10\right)$. The fact that the curves stop moving down is relevant, since it gives a clear indication that there is no advantage in introducing further complexity into the model, for no gain in model fidelity (the minimum of LF stops decreasing). Plotting different LF curves versus $n_{d}$, on the same graph, for different value of $n_{b}$, is useful to obtain a first indication for the value of $n_{d}$. Indeed, Fig. 9 shows that the minima of the curves stop decreasing for about $n_{d}=-7$. For experiments $E_{2}, E_{3}, E_{4}, E_{5}$ and $E_{6}$, the curves stop going down for $n_{d}=-8,-5,-8,-9$ and -11 respectively, suggesting a range of $-11 \leq n_{d} \leq-5$. Now that a range for $n_{d}$ is selected, the next step is to find the associated range for $n_{b}$. Fig. 10 shows $L F=L F\left(n_{a}=\right.$ $5,0 \leq n_{b} \leq 20,-11 \leq n_{d} \leq-5$ ) versus $n_{b}$, for experiment $E_{1}$. Each vertical line shows the range of LF for a specific value of $n_{b}$, with $n_{a}=5$ and $n_{d}$ taking all the possible values between -11 and -5 . In Fig. 10, it is possible to see that the LF minima stop decreasing significantly
Table 2

Identified $n_{a}, n_{b}, n_{d}$ and $n_{p}$ from experiments $E_{1}, E_{2}, E_{3}, E_{4}, E_{5}$ and $E_{6}$

\begin{tabular}{llll}
\hline$n_{a}$ & $n_{b}$ & $n_{d}$ & $n_{p}$ \\
\hline 5 & 6 & -8 & 2
\end{tabular}

for $n_{b}>4$. By applying the same procedure for experiments $E_{2}, E_{3}$, $E_{4}, E_{5}$ and $E_{6}$, the LF ceases to reduce further for $n_{b}>10,7,12,7,8$ respectively. Therefore, the range $4 \leq n_{b} \leq 12$ associated with the range $-11 \leq n_{d} \leq-5$, is suggested. It is important to underline that the objective is the identification of the simplest model structure, with a consistent $n_{a}, n_{b}$ and $n_{d}$ for the different inputs (different FSE). The use of an approximated model structure (ARX model) to describe the data, results in different suggested values of $n_{b}$ and $n_{d}$ from different experiments (however, the same $n_{a}=5$ has been identified from all experiments). The multi-step predictions of the models are calculated starting from the smallest $n_{b}=4$, and increasing in complexity until good average multi-step model performance for experiments $E_{1}, E_{2}$, $E_{3}, E_{4}, E_{5}$ and $E_{6}$, is obtained. Good results are obtained for $n_{b}=6$ (and the associated $n_{d}=-8$ ). Table 2 shows the identified values of $n_{a}$, $n_{b}$ and $n_{d}$. 


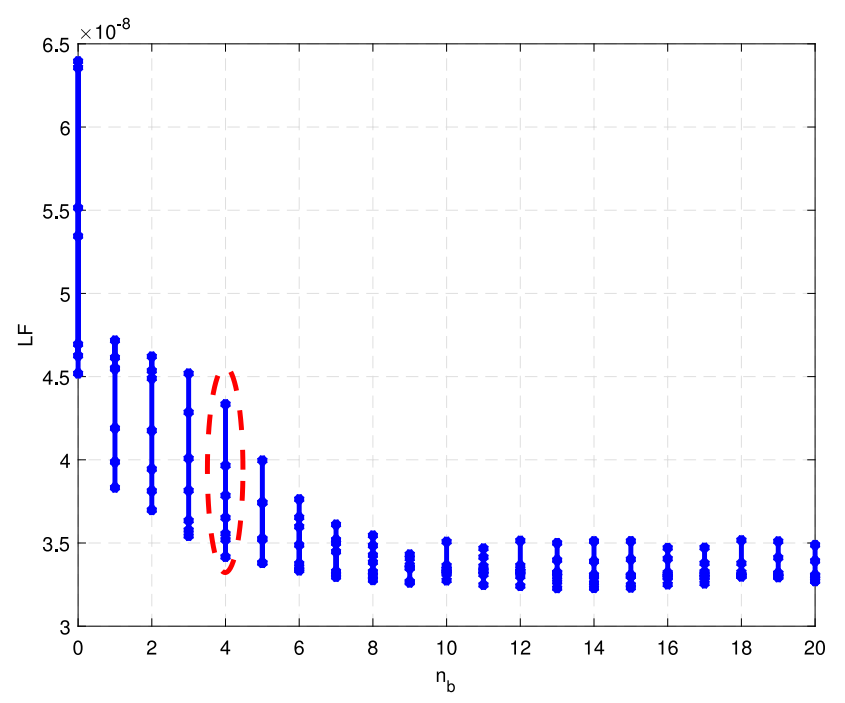

Fig. 10. Loss function for experiment $E_{1}\left(n_{a}=5,-11 \leq n_{d} \leq-5\right.$ and $\left.0 \leq n_{b} \leq 20\right)$. For this experiment, no relevant reduction of the LF for $n_{b}>4$.

\section{Validation}

The model validation step, shown in Fig. 1, is an important aspect in SI. The first model assessment is computed on the training data and, if the results are not satisfactory with the training data, the model cannot be accepted. This may be due to an incorrect model structure, which is unable to describe the complexity of the process (Nelles, 2001), or the identification algorithm may provide a parameter vector too far from the optimal solution. In a case where the performance achieved on the training data is acceptable, the real validation assessment comes when the model is tested on new data, that is, when the model has to predict the outcome of an experiment which was not used for the model parameter identification (Ljung, 2006). In order to deliver the training and validation steps, the available data is divided into separate training and validation data sets (Nelles, 2001) (Billings, 2013). Common methods utilised for model validation are the 1-step (ahead) prediction test and the multi-step (ahead) prediction test (Billings, 2013):

- 1-step (ahead) prediction test. The model output prediction, $\hat{y}$, is calculated by utilising only the process measurements $\eta$ and $y$ (no predicted past values of the output, $\hat{y}$, are used), as shown in Fig. 11(a). At each time step, the prediction error $e(k)=y(k)-\hat{y}(k)$ is computed. 1step prediction tests are often not sufficient to show model inadequacy and even models with low accuracy may generate almost perfect 1-step predictions (Billings, 2013).

- Multi-step (ahead) prediction test. The mathematical model is initialised by a few known measured output values and, successively, the model output is calculated by the previous model predicted output, $\hat{y}$, and by the given measured input, $\eta$, as shown in Fig. 11(b). Therefore, at each time step $e(k)$ may quickly accumulate.

Different error metrics can be utilised to compare the model prediction, $\hat{y}(k)$, with the measured signal, $y(k)$ (either for 1-step or multi-step prediction); in this paper, the normalised root mean-squared error (NRMSE) is utilised, defined as:

$N R M S E=\frac{\sqrt{\sum_{k}|y(k)-\hat{y}(k)|^{2}}}{\sqrt{\sum_{k}|y(k)|^{2}}}$

The NRMSE metric is selected over other metrics, such as the MSE or the mean absolute percentage error (MAPE), because the NRMSE is normalised with respect to the magnitude of $y(k)$ (unlike the MSE), and the NRMSE does not give a distorted picture of the error for $y(k)$ values close to zero (unlike the MAPE). More details about the comparison of MSE, MAPE and NRMSE can be found in (Giorgi, 2017). (a)

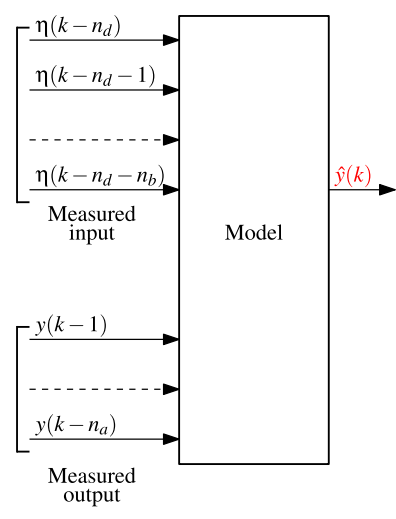

(b)

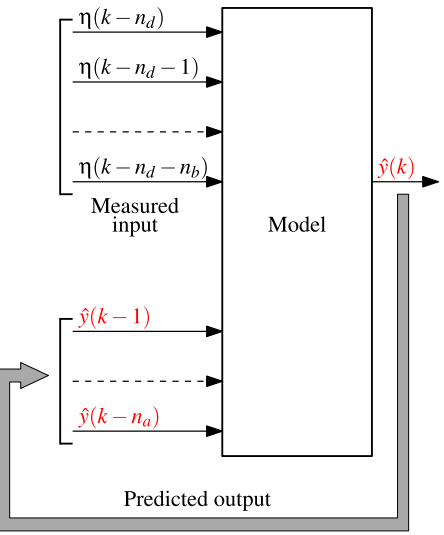

Fig. 11. (a) 1-step prediction. (b) Multi-step prediction.
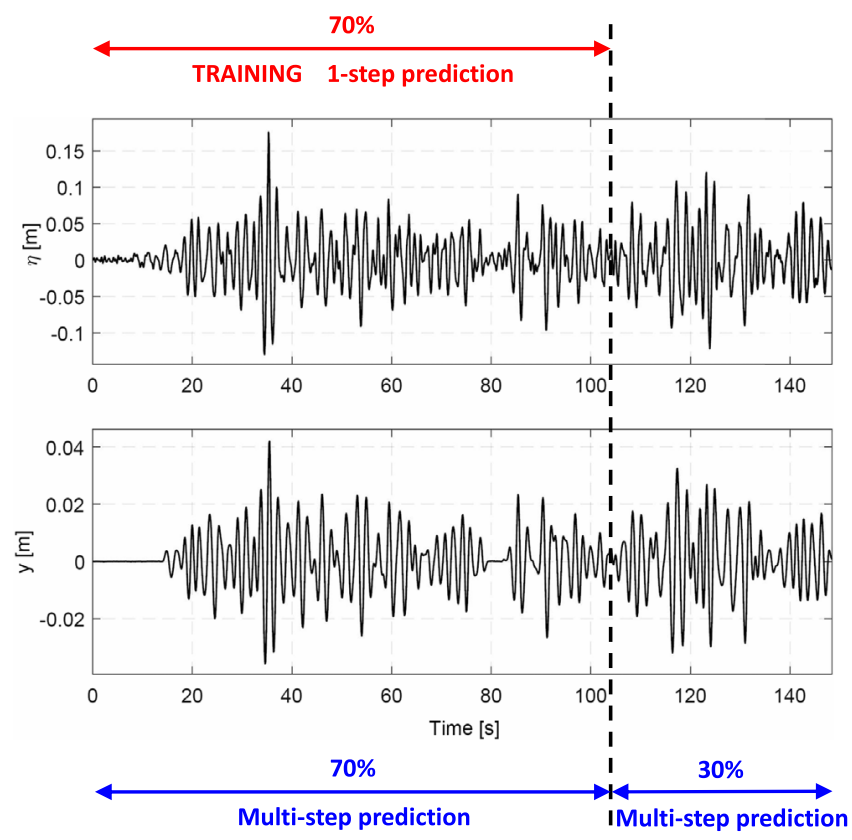

Fig. 12. In the case of single training, each experiment is divided in two parts: the first $70 \%$ time length part and the final $30 \%$ time length part (experiment $E_{1}$ is shown). Each model is trained with a 1-step prediction criterion on the first part and, once identified, the model is used to make a multi-step prediction on the same first $70 \%$ training part of the experiment, and a further multi-step prediction on the final $30 \%$ validation part of the experiment.

\section{Results}

Since ARX and KGP models are linear in the parameters, each model is trained with a 1-step prediction criterion, which leads to a convex optimisation problem (the advantageous convex optimisation would be lost by using a multi-step prediction criterion for the training). The utilised $n_{a}, n_{b}$ and $n_{d}$ values are shown in Table 2. The models have to describe the behaviour of the WEC in different sea states and, therefore, different strategies to identify the model parameters are possible, as presented in Sections 6.1 and 6.2.

\subsection{Single training model}

A first possibility to train a model, referred to here as single training, is to identify a set of parameters for each available sea state and for each model structure (in this case a total of 12 parameter sets). 


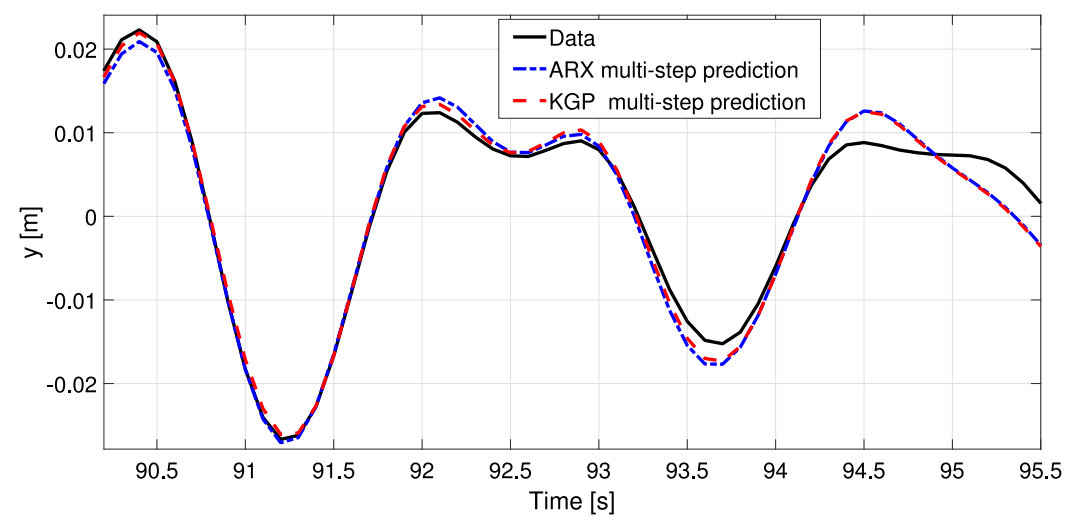

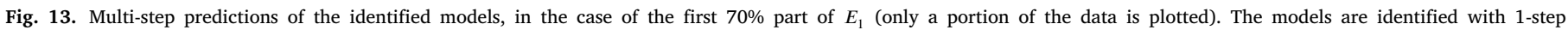
prediction single training criterion, by using the training part of $E_{1}$.

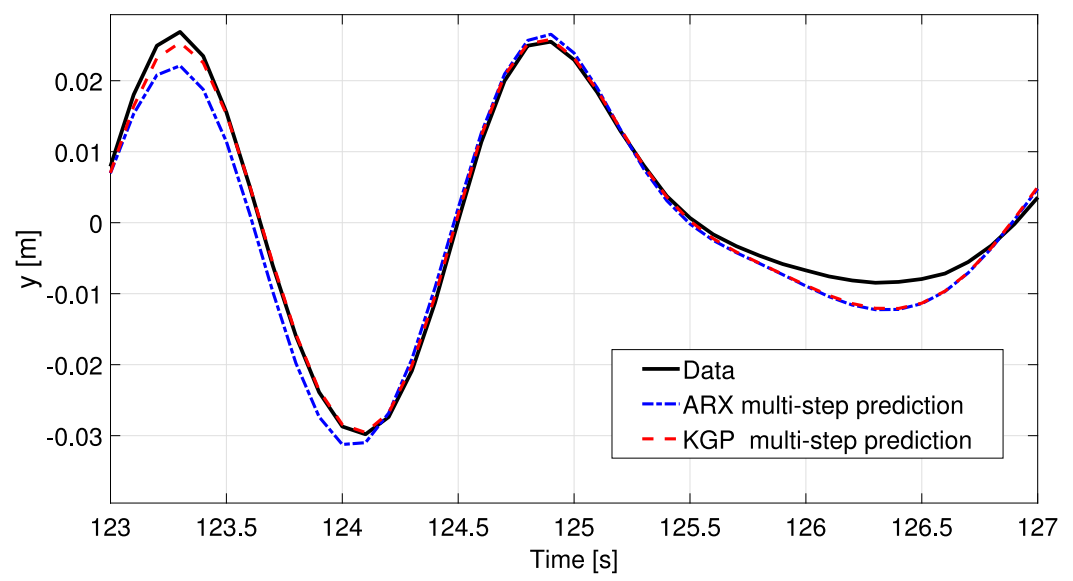

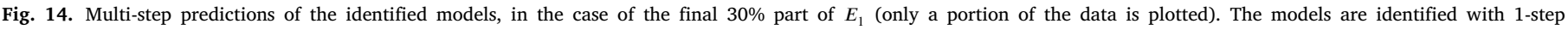
prediction single training criterion by using the training part of $E_{1}$.

Subsequently, the parameter set, corresponding to the present sea state, is loaded into the model structure to predict the behaviour of the WEC. As explained in Section 2.3, each available experiment is a realization belonging to a different sea state (see Table 1). Each experimental data set is divided in two parts: the first $70 \%$ time length part, for model training, and the final $30 \%$ time length part, for model validation (which is a common choice in system identification, data mining and machine learning (Gholami et al., 2015; Lim et al., 2000) (Ljung, 2013). Each model is trained with a 1-step prediction criterion on the first part and, once identified, the model is used to make a multi-step prediction on the same first $70 \%$ training part of the experiment, and a further multi-step prediction on the final $30 \%$ validation part of the experiment (see Fig. 12).

Table 3 shows the NRMSE multi-step prediction performance for each model, on the $70 \%$ and $30 \%$ data parts, where $\mathrm{KGP}(\mathrm{p})$ is a KGP model with maximum polynomial order $p$, as defined in Eq. (5). Furthermore, the nonlinear KGP models are compared to the linear ARX model, with a white background when the nonlinear model performs better than the linear one, and with a red background when the nonlinear model performs worse. The performance of the linear ARX models is shown with a yellow background. In each experiment (apart the final $30 \%$ of experiment $E_{2}$ ), the multi-step prediction for the KGP models, with $n_{p}=2$, is better than the multi-step prediction for the ARX models, in both the initial $70 \%$ and final $30 \%$ parts, showing that there is some nonlinearity in the data that the KGP model is able to describe. Increasing $n_{p}$ to 3 , the KGP multi-step prediction shows a degradation in validation, compared to the KGP model with $n_{p}=2$, indicating that $n_{p}=3$ introduces excess complexity into the model and suggesting overfitting. Similar conclusions apply for $n_{p}=4,5$. Therefore, the results indicate that the correct value to obtain a parsimonious KGP model is $n_{p}=2$. Table 3 shows that all the identified ARX and KGP(2) models generalise quite well. In Section 2.3 , it is supposed that the extent of hydrodynamic nonlinearity is rather limited in the utilised experiments, because of the reduced relative body displacement. This is confirmed by the fact that the NRMSE multi-step prediction of the KGP models are smaller than the ARX models, but the improvement is not significant, as shown in Table 3. Figs. 13 and 14 show the multi-step predictions of the models (identified with a 1-step prediction criterion on the first $70 \%$ part of $E_{1}$ ) in the case of the training and validation parts of $E_{1}$, respectively.

\subsection{Mixed training model}

A second possibility for model identification, referred to here as mixed training, is to obtain a unique set of parameters for each model structure, able to describe the behaviour of the WEC in any sea condition. Therefore, only one ARX model and one KGP model having $n_{p}=2$ are identified. As in the case of single training, each experiment is divided in two parts: the first $70 \%$ and the final $30 \%$. The first $70 \%$ of each dataset $E_{1}, E_{2}, E_{3}, E_{4}, E_{5}$ and $E_{6}$ are utilised together to train the models with a 1-step prediction criterion. Once the models are identified, they are validated with a multi-step prediction criterion on the final $30 \%$ part of each experiment (each model is validated in six different sea states), as shown in Fig. 15. The NRMSE multi-step prediction performance for the models are shown in Table 4, where the nonlinear KGP model is compared to the linear ARX model; with a white background when the nonlinear model performs better than 
Table 3

NRMSE models multi-step prediction performance, in the case of single training model. The nonlinear KGP models are compared to the linear ARX model, with a white background when the nonlinear model performs better than the linear one, and with a red background when the nonlinear model performs worse. The performance of the linear ARX models is shown with a yellow background.

\begin{tabular}{|c|c|c|c|c|c|c|c|c|}
\hline $\begin{array}{c}\text { Exp } \\
\text { name }\end{array}$ & $\begin{array}{l}\mathrm{Tp} \\
{[\mathrm{s}]}\end{array}$ & $\begin{array}{l}\text { Hs } \\
{[\mathrm{m}]}\end{array}$ & $\begin{array}{l}\text { Exp } \\
\text { part }\end{array}$ & $\begin{array}{c}\text { ARX } \\
\text { NRMSE }\end{array}$ & $\begin{array}{l}\text { KGP(2) } \\
\text { NRMSE }\end{array}$ & $\begin{array}{l}\text { KGP(3) } \\
\text { NRMSE }\end{array}$ & $\begin{array}{l}\text { KGP(4) } \\
\text { NRMSE }\end{array}$ & $\begin{array}{l}\text { KGP(5) } \\
\text { NRMSE }\end{array}$ \\
\hline \multirow{2}{*}{$\mathrm{E}_{1}$} & \multirow{2}{*}{2} & \multirow{2}{*}{0.124} & First 70\% & 0.1484 & 0.1461 & 0.1442 & 0.1449 & 0.1402 \\
\hline & & & Final $30 \%$ & 0.1324 & 0.1273 & 0.1276 & 0.1358 & 0.1618 \\
\hline \multirow{2}{*}{$\mathrm{E}_{2}$} & \multirow{2}{*}{2.5} & \multirow{2}{*}{0.184} & First $70 \%$ & 0.1124 & 0.1119 & 0.1064 & 0.1028 & 0.0989 \\
\hline & & & Final $30 \%$ & 0.1170 & 0.1184 & 0.1190 & 0.1217 & 0.1237 \\
\hline \multirow{2}{*}{$E_{3}$} & \multirow{2}{*}{3} & \multirow{2}{*}{0.245} & First $70 \%$ & 0.1009 & 0.0950 & 0.0923 & 0.0897 & 0.0889 \\
\hline & & & Final $30 \%$ & 0.1107 & 0.1097 & 0.1105 & 0.1079 & 0.1141 \\
\hline \multirow{2}{*}{$\mathrm{E}_{4}$} & \multirow{2}{*}{2.5} & \multirow{2}{*}{0.369} & First $70 \%$ & 0.1428 & 0.1330 & 0.1244 & 0.1223 & 0.1206 \\
\hline & & & Final $30 \%$ & 0.1377 & 0.1307 & 0.1435 & 0.1376 & 0.1621 \\
\hline \multirow{2}{*}{ E5 } & \multirow{2}{*}{3} & \multirow{2}{*}{0.490} & First $70 \%$ & 0.1433 & 0.1361 & 0.1335 & 0.1332 & 0.1314 \\
\hline & & & Final $30 \%$ & 0.1375 & 0.1263 & 0.1395 & 0.1404 & 0.1467 \\
\hline \multirow{2}{*}{ E6 } & \multirow{2}{*}{4} & \multirow{2}{*}{0.720} & First $70 \%$ & 0.1472 & 0.1401 & 0.1397 & 0.1354 & 0.1306 \\
\hline & & & Final $30 \%$ & 0.1387 & 0.1382 & 0.1407 & 0.1508 & 0.1682 \\
\hline
\end{tabular}

Table 4

Validation NRMSE models multi-step prediction performance, in the case of mixed training model. The nonlinear KGP model is compared to the linear ARX model; with a white background when the nonlinear model performs better than the linear one and with a red background when the nonlinear model performs worse. The performance of the linear ARX model is shown with a yellow background.

\begin{tabular}{|c|c|c|c|c|c|}
\hline $\begin{array}{c}\text { Exp } \\
\text { part }\end{array}$ & $\begin{array}{c}\text { Exp } \\
\text { name }\end{array}$ & $\begin{array}{c}\text { Tp } \\
{[s]}\end{array}$ & $\begin{array}{c}\text { Hs } \\
{[\mathrm{m}]}\end{array}$ & $\begin{array}{c}\text { ARX } \\
\text { NRMSE }\end{array}$ & $\begin{array}{c}\text { KGP(2) } \\
\text { NRMSE }\end{array}$ \\
\hline Final 30\% & $E_{1}$ & 2 & 0.124 & 0.1482 & 0.1413 \\
\hline Final 30\% & $E_{2}$ & 2.5 & 0.184 & 0.1160 & 0.1187 \\
\hline Final 30\% & $E_{3}$ & 3 & 0.245 & 0.1355 & 0.1362 \\
\hline Final 30\% & $E_{4}$ & 2.5 & 0.369 & 0.1388 & 0.1294 \\
\hline Final 30\% & $E_{5}$ & 3 & 0.490 & 0.1364 & 0.1251 \\
\hline Final 30\% & $E_{6}$ & 4 & 0.720 & 0.1363 & 0.1337 \\
\hline
\end{tabular}

the linear one and with a red background when the nonlinear model performs worse. The performance of the linear ARX model is shown with a yellow background. The validation of the models shows that, in experiments $E_{1}, E_{4}, E_{5}$ and $E_{6}$, the multi-step predictions of the KGP model, with $n_{p}=2$, are better than the multi-step predictions of the ARX model. Therefore, in the data there is some small nonlinearity that the KGP model is able to describe, even if the improvement is not significant.

\subsection{Single and mixed training model comparison}

At this point, the following question is posed: to obtain a good prediction for a particular sea state, is it better to train the models only on the same sea state (to tune the models only for the specific sea condition), or it is always better to train the models using all the information available, even if the extra information is from different sea states? In order to try to answer this question, the performance of the models identified with single training, shown in Table 3 , and with mixed training, shown in Table 4, are compared in Table 5, where the boxes in white indicate that the model identified with single training performs better than the model identified with mixed training trained, and in red when it performs worse. The results show that, in the case of the available experimental data, there is not a clear answer regarding the use of single or multiple sea state data.

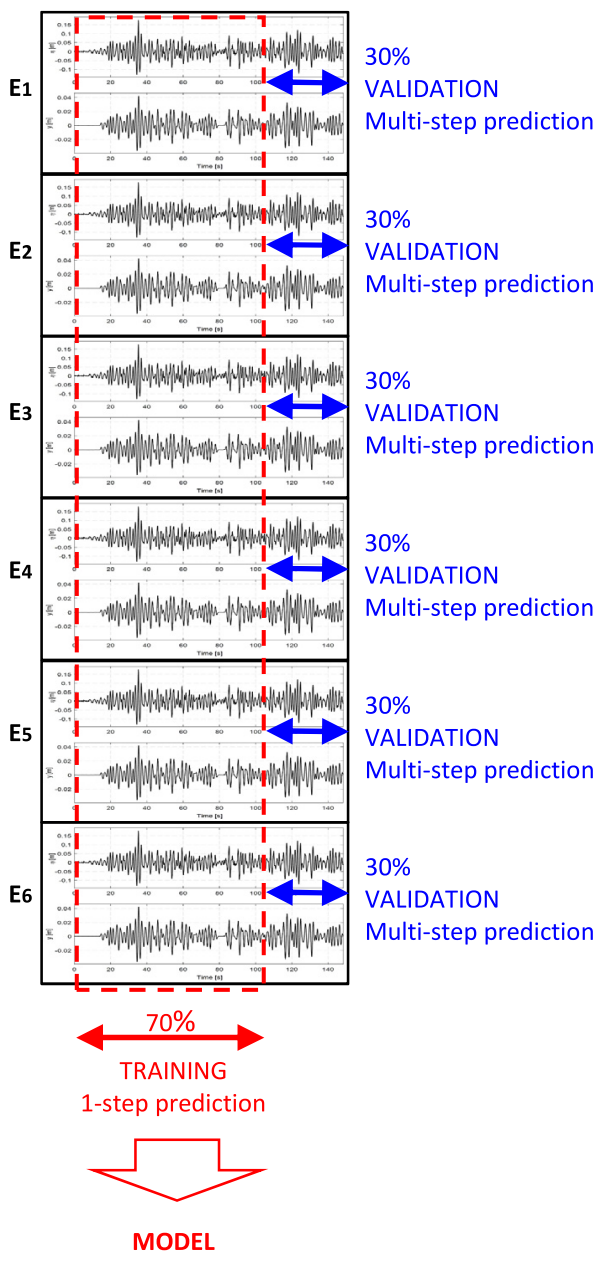

Fig. 15. In the case of mixed training, the first $70 \%$ of each dataset $E_{1}, E_{2}, E_{3}, E_{4}$, $E_{5}$ and $E_{6}$ are utilised together to train the models with a 1-step prediction criterion. Once the models are identified, they are validated with a multi-step prediction criterion on the final $30 \%$ part of each experiment (each model is validated in six different sea states). 
Table 5

Comparison of validation NRMSE models multi-step prediction performances in the cases of single and mixed training. The NRMSE values with a white background indicate that the model identified with single training performs better than the model identified with mixed training trained, and with a red background when it performs worse.

\begin{tabular}{|c|c|c|c|c|c|c|c|}
\hline \multirow[b]{2}{*}{$\begin{array}{l}\text { Exp } \\
\text { part }\end{array}$} & \multirow[b]{2}{*}{$\begin{array}{c}\text { Exp } \\
\text { name }\end{array}$} & \multirow[b]{2}{*}{$\begin{array}{l}\text { Tp } \\
{[s]}\end{array}$} & \multirow[b]{2}{*}{$\begin{array}{c}\text { Hs } \\
{[\mathrm{m}]}\end{array}$} & \multicolumn{2}{|c|}{ Single training } & \multicolumn{2}{|c|}{ Mixed training } \\
\hline & & & & $\begin{array}{c}\text { ARX } \\
\text { NRMSE }\end{array}$ & $\begin{array}{l}\text { KGP(2) } \\
\text { NRMSE }\end{array}$ & $\begin{array}{c}\text { ARX } \\
\text { NRMSE }\end{array}$ & $\begin{array}{l}\text { KGP(2) } \\
\text { NRMSE }\end{array}$ \\
\hline Final $30 \%$ & $E_{1}$ & 2 & 0.124 & 0.1324 & 0.1273 & 0.1482 & 0.1413 \\
\hline Final $30 \%$ & $E_{2}$ & 2.5 & 0.184 & 0.1170 & 0.1184 & 0.1160 & 0.1187 \\
\hline Final $30 \%$ & $E_{3}$ & 3 & 0.245 & 0.1107 & 0.1097 & 0.1355 & 0.1362 \\
\hline Final $30 \%$ & $\mathrm{E}_{4}$ & 2.5 & 0.369 & 0.1377 & 0.1307 & 0.1388 & 0.1294 \\
\hline Final $30 \%$ & $E_{5}$ & 3 & 0.490 & 0.1375 & 0.1263 & 0.1364 & 0.1251 \\
\hline Final $30 \%$ & $\mathrm{E}_{6}$ & 4 & 0.720 & 0.1387 & 0.1382 & 0.1363 & 0.1337 \\
\hline
\end{tabular}

Table 6

Validation NRMSE models multi-step prediction performances, in the case of interpolation/extrapolation model ability. The nonlinear KGP models are compared to the linear ARX model, with a white background when the nonlinear model performs better than the linear one, and with a red background when the nonlinear model performs worse. The performance of the linear ARX models is shown with a yellow background.

\begin{tabular}{|c|c|c|c|c|}
\hline $\begin{array}{c}\text { Validation } \\
\text { experiment }\end{array}$ & $\begin{array}{c}\text { Tp } \\
{[\mathbf{s}]}\end{array}$ & $\begin{array}{c}\text { Hs } \\
{[\mathrm{m}]}\end{array}$ & $\begin{array}{c}\text { ARX } \\
\text { NRMSE }\end{array}$ & $\begin{array}{c}\text { KGP(2) } \\
\text { NRMSE }\end{array}$ \\
\hline$E_{1}$ & 2 & 0.124 & 0.1674 & 0.1607 \\
\hline$E_{2}$ & 2.5 & 0.184 & 0.1172 & 0.1186 \\
\hline$E_{3}$ & 3 & 0.245 & 0.1528 & 0.1526 \\
\hline$E_{4}$ & 2.5 & 0.369 & 0.1463 & 0.1366 \\
\hline$E_{5}$ & 3 & 0.490 & 0.1418 & 0.1374 \\
\hline$E_{6}$ & 4 & 0.720 & 0.1411 & 0.1340 \\
\hline
\end{tabular}

\subsection{Model interpolation/extrapolation ability}

The space of sea states, described in terms of $H_{s}$ and $T_{p}$, is usually discretised by selecting a finite number of $H_{s}$ and $T_{p}$ values. The level of discretisation is a compromise between the total number of sea states and the accuracy of each sea state description. The identification of models with a larger number of sea states requires more experiments and, consequently, a larger number of model parameter sets to interpolate between. Therefore, the number of utilised sea states should be limited, and the models should be able to describe any sea condition, by interpolating/extrapolating the information, provided from the known sea states. In the case of the present scaled Wavestar WEC, six different experiments are available from six different sea states.

In order to test the ability of the identified models, in interpolating/extrapolating the data, each model is trained with a 1-step prediction criterion, by simultaneously using $100 \%$ of five experiments and, once identified, is validated with a multi-step prediction measure on $100 \%$ of the sixth missing experiment. In total, the procedure has been repeated six times, each time by changing the sixth validating experiment, as shown in Table 6, which shows that the NRMSE multi-step prediction model performance is marginally poorer than the NRMSE multi-step prediction model performance in Table 4, (where the models are trained using the first $70 \%$ of all six experiments). The results indicate a good ability of the models to interpolate/extrapolate the information provided from the known sea states to predict an unknown sea condition. Also, in this case, in each experiment (apart from experiment $E_{2}$ ), the multi-step prediction for the KGP models with $n_{p}=2$ is better than the multi-step prediction for the ARX models.

\section{Conclusions}

The framework for hydrodynamic discrete-time model identification (already applied to numerical wave tank data in previous work (Giorgi, 2017; Giorgi et al., 2016a) (Davidson et al., 2016, 2015) (Ringwood et al., 2016) in this paper, is successfully broadened to the context of experimental data, gathered at the COAST Laboratory, where hydrodynamic tests were carried out on a scaled Wavestar wave energy converter point-absorber, attached to a hydraulic PTO, having a control strategy described by Eq. (1). Since both linear and nonlinear models employed present a 'linear in the parameters' identification problem, they are assured of achieving global fitting error minima, facilitating a consistency of comparison. Furthermore, this research showed that the models employed are computationally simple and fast to identify and run.

For the tank tests considered, the device essentially behaves as a wave follower (due to the PTO setting and control strategy utilised in the tank experiments), and little benefit of the nonlinear model (over the linear) is evident. As a result, the appropriate nonlinear polynomial order is a modest 2 . One notable issue, in the determination of the appropriate dynamical model orders, is the lack of clear indication, compared to the case for numerical tank data. This is, no doubt due to the added presence of measurement noise and other parasitic effects. Nevertheless, reliable models are identified with encouraging values of model fit. The use of multiple data sets helps in the consistent estimation of model structure and parameter identification.

An interesting potential future study would involve the use of a different PTO control strategy on the same Wavestar device, which would create a more significant relative body displacement and/or velocity and introduce, in this way, more nonlinear hydrodynamic effects into the system. However, the same identification framework, presented in this work, could be employed, though with the possible result of ARX and KGP models having different dynamical and maximum polynomial orders to adapt to the behaviour of the new WEC-PTO system. With such new data, the nonlinear KGP model is likely to show a superiority compared to the ARX model, as evidenced in (Giorgi et al., 2016a).

\section{Acknowledgements}

This paper is based upon work supported by Science Foundation Ireland [Grant No. 13/IA/1886] and by Enterprise Ireland [Grant $\mathrm{EI} / \mathrm{CF} / 2011 / 1320]$.

\section{References}

Armesto, J.A., Guanche, R., Iturrioz, A., Vidal, C., Losada, I.J., 2014. Identification of state-space coefficients for oscillating water columns using temporal series. Ocean Eng. 79, 43-49. 
Atluri, S., Magee, A., Lambrakos, K., 2009. CFD as a design tool for hydrodynamic loading on offshore structures. In: Proceedings of the 28th International Conference on Ocean, Offshore and Arctic Engineering. American Society of Mechanical Engineers, pp. 499-507.

Bacelli, G., Coe, R.G., Patterson, D., Wilson, D., 2017. System identification of a heaving point absorber: Design of experiment and device modeling. Energies 10 (4), 472.

Bhinder, M.A., 2013. 3D Non-linear Numerical Hydrodynamic Modelling of Floating Wave Energy Converters (Ph.D. thesis). Ecole Centrale de Nantes.

Bhinder, M.A., Babarit, A., Gentaz, L., Ferrant, P., 2011. Assessment of viscous damping via $3 \mathrm{D}-\mathrm{CFD}$ modelling of a floating wave energy device. In: Proceedings of the 9th European Wave and Tidal Energy Conference.

Bhinder, M.A., Babarit, A., Gentaz, L., Ferrant, P., 2012. Effect of viscous forces on the performance of a surging wave energy converter. In: Proceedings of ISOPE2012, pp. 545-549.

Billings, S., 2013. Nonlinear System Identification: NARMAX Methods in the Time, Frequency, and Spatio-Temporal Domains. Wiley.

Box, G., Jenkins, G., Gregory, R., 1994. Time Series Analysis, Forecasting and Control. Prentice Hall, Upper Saddle River, NJ, USA.

Burnham, K., Anderson, D., 2013. Model Selection and Inference: A Practical Information-Theoretic Approach. Springer New York.

Chetouani, Y., 2008. Using ARX approach for modelling and prediction of the dynamics of a reactor-exchanger. In: Proceedings of the Institution of Chemical Engineers Symposium Series, vol. 154, pp. 1-9.

Cho, H., Bacelli, G., Coe, R.G., 2018. Linear and nonlinear system identification of a wave energy converter. In: Proceedings of the Waterpower Week in Washington.

COAST, 2018. Coastal ocean and sediment transport laboratory. https://www.plymouth. ac.uk/research/institutes/marine-institute/coast-laboratory, (Accessed 30 October 2018).

Coe, R.G., Bacelli, G., Patterson, D., Wilson, D.G., 2016. Advanced WEC Dynamics \& Controls, FY16 Testing Report. Technical Report SAND2016-10094, Sandia National Laboratories.

Davidson, J., Giorgi, S., Ringwood, J.V., 2015. Linear parametric hydrodynamic models for ocean wave energy converters identified from numerical wave tank experiments. Ocean Eng. 103, 31-39.

Davidson, J., Giorgi, S., Ringwood, J.V., 2016. Identification of wave energy device models from numerical wave tank data - Part 1: Numerical wave tank identification tests. IEEE Trans. Sustain. Energy 7 (3), 1012-1019.

Draper, N., Smith, H., 1998. Applied regression analysis. In: Wiley Series in Probability and Statistics: Texts and References Section, (v. 1), Wiley.

Falnes, J., 1995. On non-causal impulse response functions related to propagating water waves. Appl. Ocean Res. 17 (6), 379-389.

Falnes, J., 2002. Ocean Waves and Oscillating Systems, Linear Interactions Including Wave-Energy Extraction. Cambridge University Press.

Folley, M., Whittaker, T., Vant Hoff, J., 2007. The design of small seabed-mounted bottom-hinged wave energy converters. In: Proceedings of the 7th European Wave and Tidal Energy Conference.

Gholami, V., Chau, K., Fadaee, F., Torkaman, J., Ghaffari, A., 2015. Modeling of groundwater level fluctuations using dendrochronology in alluvial aquifers. J. Hydrol. 529, 1060-1069.

Giorgi, S., 2017. Linear and nonlinear parametric hydrodynamic models for wave energy converters identified from recorded data. (Ph.D. thesis). Maynooth University.

Giorgi, S., Davidson, J., Ringwood, J.V., 2016a. Identification of wave energy device models from numerical wave tank data - Part 2: Data-based model determination. IEEE Trans. Sustain. Energy 7 (3), 1020-1027.

Giorgi, G., Retes, M.P., Ringwood, J.V., 2016b. Nonlinear hydrodynamic models for heaving buoy wave energy converters. In: Proceedings of the 3rd Asian Wave and Tidal Energy Conference.
Giorgi, G., Ringwood, J.V., 2016. Computationally efficient nonlinear Froude-Krylov force calculations for heaving axisymmetric wave energy point absorbers. J. Ocean Eng. Mar. Energy 1 (3), 21-33.

Giorgi, G., Ringwood, V.J., 2017. Consistency of viscous drag identification tests for wave energy applications. In: Proceedings of the 12th European Wave and Tidal Energy Conference.

Golub, G., Van Loan, C., 2012. Matrix computations, fourth ed. In: Matrix Computations, Johns Hopkins University Press, Baltimore, Maryland, USA.

Hansen, R.H., Kramer, M.M., 2011. Modelling and control of the Wavestar prototype. In: Proceedings of the 9th European Wave and Tidal Energy Conference.

Isermann, R., Mnchhof, M., 2011. Identification of Dynamic Systems, An Introduction with Applications. Springer-Verlag Berlin Heidelberg.

Jakobsen, M., 2014. Technical Report on Wave-Structure Interactions on Point Absorber. Technical Report, Aalborg University.

Jakobsen, M., 2015. Wave-Structure Interactions on Point Absorbers - An Experimental Study (Ph.D. thesis). Aalborg University.

Jakobsen, M.M., Beatty, S., Iglesias, G., Kramer, M.M., 2016. Characterization of loads on a hemispherical point absorber wave energy converter. Int. J. Mar. Energy 13, $1-15$.

Keesman, K.J., 2011. System Identification An Introduction. Springer.

Lim, T.-S., Loh, W.-Y., Shih, Y.-S., 2000. A comparison of prediction accuracy, complexity, and training time of thirty-three old and new classification algorithms. Mach. Learn. 40 (3), 203-229.

Ljung, L., 1999. System Identification: Theory for the User, second ed. Prentice Hall PTR, Upper Saddle River, NJ, USA.

Ljung, L., 2006. Some aspects on nonlinear system identification. IFAC Proc. 39 (1), 110-121.

Ljung, L., 2013. MATLAB: System Identification Toolbox, User's Guide. MathWorks, Natick, Massachusetts, USA.

Nelles, O., 2001. Nonlinear System Identification: From Classical Approaches to Neural Networks and Fuzzy Models. Springer, Berlin Heidelberg, Germany.

Norton, J., 2009. An introduction to identification. In: Dover Books on Electrical Engineering Series, Dover Publications.

Norton, J., Walter, E., Pronzato, L., 2010. Identification of Parametric Models from Experimental Data. In: Communications and Control Engineering, Springer London.

Paparella, F., Bacelli, G., Paulmeno, A., Mouring, S.E., Ringwood, J.V., 2016. Multibody modelling of wave energy converters using pseudo-spectral methods with application to a three-body hinge-barge device. IEEE Trans. Sustain. Energy 7 (3), 966-974.

Penalba, M., Giorgi, G., Ringwood, J.V., 2017. Mathematical modelling of wave energy converters: a review of nonlinear approaches. Renew. Sustain. Energy Rev. 78, 1188-1207.

Pintelon, R., Schoukens, J., 2012. System Identification: A Frequency Domain Approach. Wiley.

Ringwood, J., Austin, P., Monteith, W., 1993. Forecasting weekly electricity consumption: A case study. Energy Econ. 15 (4), 285-296.

Ringwood, J.V., Davidson, J., Giorgi, S., 2016. In: Folley, M. (Ed.), Numerical Modelling of Wave Energy Converters: State-of-the-Art Techniques for Single Devices and Arrays (Chapter 7: Identifying Models Using Recorded Data). Elsevier Science \& Technology Books, London, UK, pp. 123-147.

Sauer, T., 2012. Numerical Analysis. Pearson Education, NY, USA.

Soderstrom, T., Stoica, P., 1989. System Identification. Prentice Hall, Upper Saddle River, NJ, USA.

Wolgamot, H.A., Fitzgerald, C.J., 2015. Nonlinear hydrodynamic and real fluid effects on wave energy converters. Proc. Inst. Mech. Eng. A 1-23.

Zurkinden, A.S., Ferri, F., Beatty, S., Kofoed, J.P., Kramer, M., 2014. Non-linear numerical modeling and experimental testing of a point absorber wave energy converter. Ocean Eng. 78, 11-21. 